\title{
El ecoturismo en las reservas de la biósfera: Prácticas y actitudes hacia la conservación
}

\author{
Kennedy Obombo Magio* \\ Consejo Nacional de Ciencia y Tecnología - CONACYT (México)
}

Mónica Velarde Valdez**

Universidad de Occidente(México)

\begin{abstract}
Resumen: El ecoturismo se ha reconocido como una opción viable para integrar la conservación y el desarrollo socioeconómico en las Áreas Naturales Protegidas. Sin embargo, es importante realizar investigaciones empíricas que comprueben la viabilidad economica y la sustentabilidad ecológica del concepto. El objetivo del estudio fue caracterizar al ecoturismo como una estrategia de conservación y desarrollo sustentable en las reservas de la biósfera. Para ello, se analiza la relación entre los beneficios y las actitudes de las comunidades locales hacia la conservación. Se aplicaron métodos mixtos, incluyen, una encuesta cuantitativa ( $\mathrm{n}=138)$, entrevistas abiertas $(n=22)$ y cuatro grupos focales. Se encontró que el ecoturismo puede generar importantes beneficios económicos para las comunidades locales e incentivar su participación en iniciativas de conservación. También, se encontró que los incentivos económicos directos del ecoturismo por sí solos no son suficientes para transformar las actitudes y prácticas de los residentes a favor de la conservación.
\end{abstract}

Palabras Clave: Ecoturismo; Conservación; Desarrollo sustentable; Reservas de la biósfera; Los Tuxtlas, México; Maasai Mara, Kenia.

\section{Ecotourism in biosphere reserves: Practices and attitudes towards conservation?}

Abstract: Ecotourism has emerged as a viable option for integrating conservation and socio-economic development in protected areas. However, it is important to carry out empirical studies that provide evidence of its economic viability and environmental sustainability. The primary objective of this study was to characterise ecotourism as a strategy for conservation and sustainable development in Biosphere Reserves. To achieve this, we analysed the relationship between the potential benefits and the attitudes of local communities towards conservation. A mix of methods was used, including a quantitative survey ( $\mathrm{n}=138$ ), open interviews $(\mathrm{n}=22)$ and four focal group discussions. The results show that ecotourism has potential for generating significant economic benefits to the local communities and for encouraging their participation in conservation initiatives. They also revealed that direct economic incentives are not sufficient to change residents' attitudes and practices in favour of conservation.

Keywords: Ecotourism; Conservation; Sustainable developmen; Biosphere Reserves; Los Tuxtlas, Mexico; Maasai Mara, Kenya.

\section{Introducción}

En los últimos años el ecoturismo se ha presentado como una opción viable tanto para conservar el patrimonio natural (Rudzewicz y Lanzar, 2008) y cultural (De La Rosa, 2003), como para promover un desarrollo sustentable en las Áreas Naturales Protegidas (ANPs) (Vargas del Río y Brenner, 2013).

\footnotetext{
* Profesor Investigador del Programa Cátedras de CONACYT comisionado al Tecnológico Nacional de México/Instituto Tecnológico de Cancún, División de Estudios de Posgrado e Investigación, Proyecto 1071: Estudios de Turismo y Sustentabilidad en el Caribe Mexicano; E-mail: magiobom@yahoo.com

** Profesor Investigador del Doctorado en Gestión del Turismo - DGT; E-mail: mvelardemx@yahoo.com
} 
Por lo tanto, el análisis de la relación turismo-conservación en estas unidades de conservación es de gran importancia, dado que las distintas fases de consumo de un producto turístico guardan relación con el medio ambiente (Budowski, 1976).

El ecoturismo surge de la necesidad de integrar la conservación con el desarrollo socioeconómico de las comunidades adyacentes a las áreas naturales protegidas (Goodwin, 1996 y 2007). Vargas del Río y Brenner (2013) coincide en que los proyectos de ecoturismo comunitario han aparecido ante todo en áreas naturales, legalmente protegidas o indefensas, que poseen altos niveles de biodiversidad imputables al conocimiento ancestral y manejo cuidadoso de los pobladores locales y con cierto potencial de mercado al estar ubicados cerca de centros turísticos establecidos. Sin embargo, se ha cuestionado la sustentabilidad ecológica y la viabilidad económica del ecoturismo como una estrategia de conservación en estas unidades de conservación, por lo que es importante realizar investigaciones empíricas relacionadas con estos aspectos.

Por ejemplo, se ha criticado la manera en que se han creado y se operan algunas Áreas Naturales Protegidas y se señala la falta de participación local como uno de los principales retos que enfrentan en su gestión (véanse los trabajos de Hvenegaard, 1994; Vargas del Río \& Brenner, 2013). Esto genera las siguientes preguntas fundamentales: ¿Para quién se crean estas unidades de conservación y a qué costo? Desdoblando este cuestionamiento, se señala que cualquier estilo de gestión ambiental que ignora la participación de las comunidades locales y les niega beneficios directos e indirectos, no contribuye a los principios de la sustentabilidad y está destinado a fracasar inevitablemente (Magio, Velarde y Santillán, 2013).

De igual forma, el problema de la participación comunitaria se aborda en el Programa sobre el Hombre y la Biósfera, denominado en inglés como Man and Biosphere-MAB, y se observa que en la mayoría de las reservas de la biósfera que forman parte de este programa, no se ha podido lograr la integración de las iniciativas de conservación y desarrollo local y asegurar una amplia participación de las comunidades locales (Van Cuong, Dart y Hockings, 2017). Kinker (2002:87-105), haciendo referencia a experiencias en parques nacionales de América Latina, África y Asia, concluye que:

...no es posible conservar y proteger los recursos naturales, especialmente en los países en vías de desarrollo, excluyendo la planificación de las necesidades humanas de las comunidades locales.

Por el contrario, enfatiza que:

...el principal motivo del énfasis de la población local en el desarrollo del ecoturismo en áreas protegidas, es estructurarlo para que satisfaga las necesidades de la comunidad aliviando la presión antrópica que amenaza esas áreas (p. 90).

Para plantear el problema de la presente investigación, se señala que la ejecución del ecoturismo en las reservas de la biósfera bajo el Programa sobre el Hombre y la Biósfera no ha logrado integrar la conservación y el desarrollo socioeconómico, por lo que existe una necesidad de cambiar el enfoque de implementación. En el Programa, el desarrollo socioeconómico sirve como un incentivo para influir en las actitudes de las comunidades locales hacia el medioambiente natural y motivar su participación en iniciativas de conservación (IUCN, 1994); ya que se generan actitudes negativas cuando los beneficios económicos directos son mínimos, lo cual se convierte en un reto de gestión ambiental.

Sin embargo, se ha cuestionado esta ideología sostenida por la UNESCO de que las comunidades adyacentes a las unidades de conservación automáticamente abandonarán sus prácticas destructivas cuando tengan un ingreso alternativo (Fennel, 2014). De aquí surge la siguiente pregunta: ¿es simplemente una cuestión de ingresos o hay otros factores que influyen en las actitudes hacia la conservación, como por ejemplo, el empoderamiento de la comunidad local, su participación activa, las estructuras de propiedad de los proyectos de desarrollo, las cuestiones legales, la relación entre los actores, así como los factores sociodemográficos?

Para aclarar y responder estos cuestionamientos, tanto los académicos como los políticos están proponiendo investigaciones que mejoren la eficacia de las estrategias de conservación a través del desarrollo local (véase por ejemplo Honey, 2008; Stronza \& Durham, 2008; Eric et al., 2011; Brenner \& Job, 2012; Vargas del Río \& Brenner, 2013). Además, Guerrero Rodríguez (2010:43) señala que es evidente "la falta de investigación acerca de las realidades existentes en los proyectos de ecoturismo", por lo que sugiere indagar sobre las diferentes estrategias empleadas en la implementación del concepto y evaluar si los proyectos mantienen una orientación hacia la conservación de los recursos naturales y si consideran la sustentabilidad social y cultural en los espacios donde se desarrolla. 
El presente estudio tuvo dos objetivos principales, primero, caracterizar el ecoturismo como una estrategia de conservación. Segundo, analizar la relación entre los beneficios del ecoturismo y las actitudes que tienen las comunidades involucradas hacia la conservación. Se plantearon tres hipótesis: 1. Los incentivos económicos por sí solos no son suficientes para transformar las actitudes y prácticas de las comunidades sobre la conservación; 2. Una mayor participación de las comunidades locales y la distribución equitativa de los costos y beneficios pueden generar actitudes y prácticas positivas que apoyan a la conservación; y 3. El ecoturismo puede influir negativamente en la conservación como resultado de acciones involuntarias o equivocadas.

La investigación se desarrolló en cuatro grupos ejidales: los ejidos Adolfo López Mateos y Ruiz Cortines, ambos ubicados en la reserva de la biósfera de Los Tuxtlas-RBLT, en el estado de Veracruz, México, y los ejidos Koiyaki (Koiyaki Group Ranch) y Lemek/Ol Chorro (Lemek Group Ranch) en la reserva de la biósfera de Maasai Mara-RBMM, en Kenia. Las cuatro comunidades de estos grupos ejidales se encuentran involucradas en las iniciativas de conservación y recientemente se han visto impactadas por proyectos ecoturísticos. En cuanto al alcance y profundidad, cabe aclarar que el presente es un estudio exploratorio cuyos resultados y conclusiones no pueden llegar a convertirse en generalizaciones; sin embargo, el análisis y la discusión aportan conocimientos teóricos y empíricos para el desarrollo futuro del ecoturismo, que integre la conservación y el desarrollo socioeconómico de las comunidades locales.

\section{Ecoturismo, conservación y desarrollo local en las reservas de la biósfera}

Los términos conservación y sustentabilidad se han convertido en términos populares y de moda para la gestión de los recursos naturales (Gómez, 2011). De manera igual, el origen y la adopción del concepto "ecoturismo" se ha vinculado a la evolución semántica de los dos términos. Por un lado, el desarrollo sustentable surgió durante la década de los 80 como el enlace integral e inevitable entre el sistema natural y el desarrollo (Cardoso-Jiménez, 2006). Cardoso-Jiménez (2006: 7) señala que "Se refiere a un repetitivo proceso de cambio en el cual la explotación de los recursos naturales, la dirección de la inversión y del progreso científico y tecnológico, junto con el cambio institucional, permita satisfacer las necesidades sociales presentes y futuras".

Sin embargo, su implementación ha sido un reto muy serio sobre todo para los países en vías de desarrollo. Actualmente, es difícil hablar de la sustentabilidad turística con las desigualdades inherentes al funcionamiento práctico del sector (Jamal \& Camargo, 2014), un continuo crecimiento de impactos negativos del turismo en las áreas naturales protegidas (Laven, Wall-Reinius y Fredman., 2015), la degradación ecológica y el agotamiento de los recursos naturales (Pérez Villegas \& Carrascal, 2000) y la pérdida de playas públicas (Mc Coy Cador \& Sosa Ferreira, 2016) en destinos turísticos costeros.

Por otro lado, Blamey (2001) describe la evolución del término "conservación" a través de dos etapas: la primera se basa en la idea de que la conservación debe implicar el mantenimiento de la armonía entre la humanidad y la naturaleza, y la segunda, en que la conservación se relaciona con el uso eficiente de los recursos, y la percepción final fue que la conservación y la preservación podrían idealmente ser abordadas desde el punto de vista de la religión y la espiritualidad. La idea de integrar el turismo con la conservación, probablemente ha existido desde la creación de los parques nacionales en el siglo XIX (Adams \& McShane, 1992).

Es evidente que, a fin de evitar o al menos minimizar los efectos adversos y aprovechar al máximo los beneficios potenciales del turismo en áreas naturales, se requiere de un enfoque más efectivo y ambientalmente responsable a nivel mundial. Este nuevo enfoque se conoce ya universalmente como turismo ecológico o ecoturismo. No hay un acuerdo general sobre quién inventó o utilizó por primera vez el término "ecoturismo", sólo se infiere que en el año 1976, Budowski (1976) utilizó los principios de ecoturismo para describir sus pensamientos en sus trabajos, que vinculan conservación con desarrollo socioeconómico, aunque no mencionó la palabra ecoturismo. El término apareció por primera vez en una publicación realizada durante los años 80 (Blamey, 2001). Ceballos Lascuráin, a quien se le atribuye el mérito de haber sido el primero en utilizar el término ecoturismo en 1980, definió el término así:

Es aquella modalidad turística ambientalmente responsable consistente en viajar o visitar áreas naturales [...] con el fin de disfrutar, apreciar y estudiar los atractivos naturales (paisaje, flora y fauna silvestres) de dichas áreas, así como cualquier manifestación cultural (del presente y del pasado) que puedan encontrarse ahí, a través de un proceso que promueve la conservación, tiene bajo impacto ambiental y cultural y [...] constituye un beneficio social y económico para las poblaciones locales. (Ceballos Lascuráin, 1996: p. 150)ํ․ 
Lo anterior indica que la definición de ecoturismo comprende un componente normativo; sólo a través del establecimiento de lineamientos estrictos y de su cumplimiento, se podrá garantizar que el ecoturismo no se convierta en un agente dañino para el patrimonio natural o cultural de un país o región. Björk (2007: 25) considera que "la conceptualización y la operacionalización del ecoturismo obstaculizan su implementación". Según él, existen numerosas definiciones del concepto por lo que el problema principal no es la falta de una mejor definición, sino cómo derivar principios, directrices y criterios pertinentes y adecuados para implementar diferentes proyectos ecoturisticos en diversos contextos. El componente económico hace énfasis en el trabajo productivo, ingreso, satisfacción racional de necesidades legítimas, suficiencia y calidad de bienes públicos. En el componente social, se integran las propuestas para promover condiciones de creciente igualdad, efectiva igualdad de oportunidades, convivencia y justicia social (Blamey, 2001).

Tomando en cuenta los planteamientos de IUCN (1994), UNESCO (1995) y Alcamo y Bennett (2003), se resalta que el ecoturismo en las áreas naturales protegidas puede contribuir al desarrollo socioeconómico, y servir como incentivo para que la comunidad local participe en iniciativas de conservación. Cuando los beneficios son mínimos, se generan actitudes negativas, lo que se convierte en un reto de gestión ambiental en las áreas naturales protegidas. En el siguiente marco conceptual, se presenta una relación precisa entre el desarrollo socioeconómico y las actitudes hacia la conservación (veáse Figura 1). Como se observa, las actividades turísticas en las ANP pueden generar dos tipos de actitudes hacia la conservación: positivas o negativas, dando lugar al éxito o al fracaso en su gestión.

\section{Figura 1: Turismo y las actitudes hacia la conservación en las Áreas Naturales Protegidas (ANPs)}

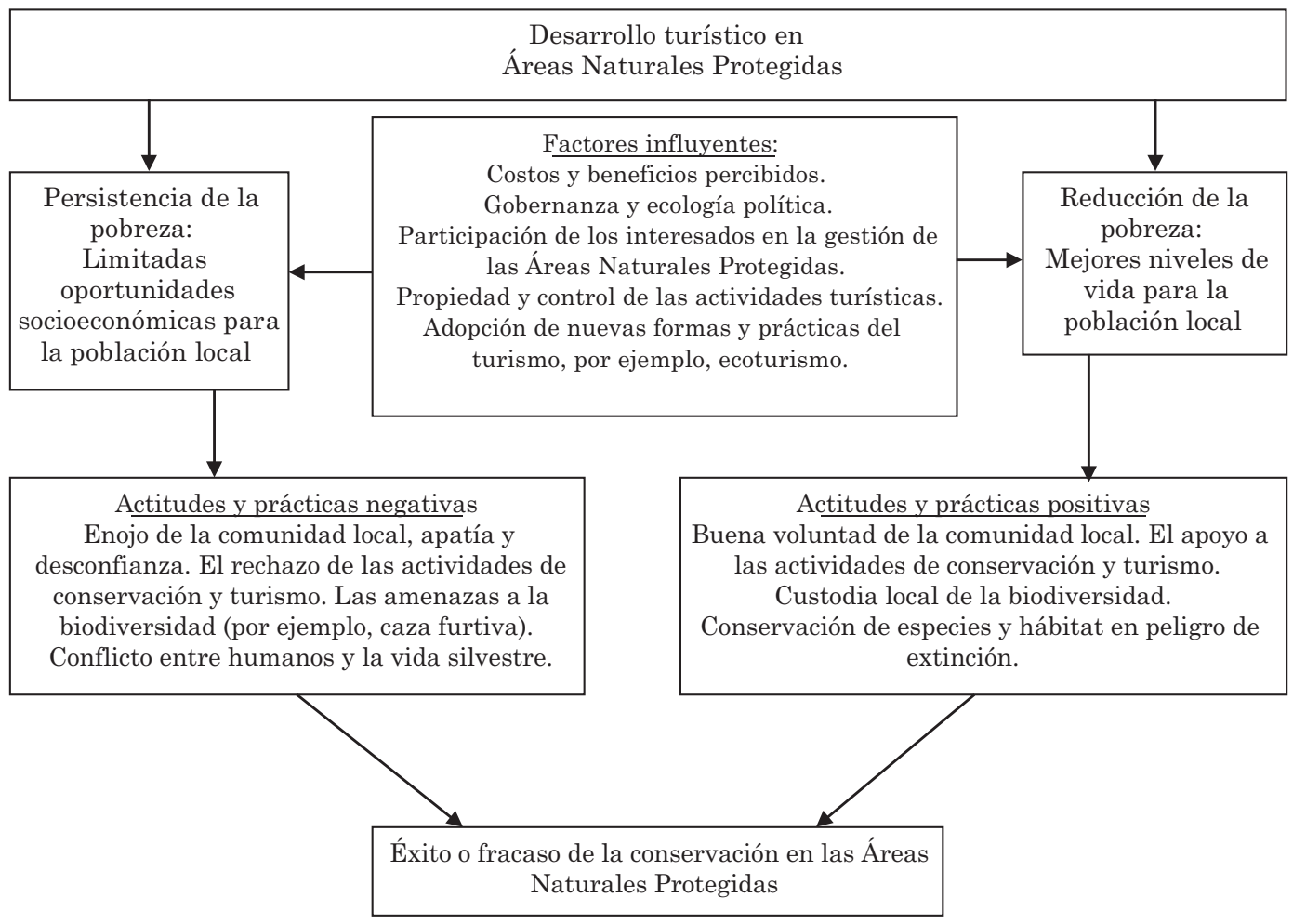

Fuente: Elaboración propia 
Una de las estrategias de conservación y desarrollo sustentable que se ha aplicado cada vez más durante las últimas cuatro décadas, es el programa sobre el Hombre y la Biósfera (MAB, por sus siglas en inglés) de la UNESCO. Es una iniciativa interdisciplinaria de investigación medioambiental que se inició en 1972 con la finalidad de establecer la base, dentro de las ciencias naturales y sociales, para la utilización racional y la conservación de los recursos de la biósfera y para mejorar la relación global entre las personas y el medio ambiente (Batisse, 1980 y 1982). En la ejecución del programa, se destaca el concepto de las reservas de la biósfera, que es una sub-categoría del grupo VI de las áreas naturales protegidas reconocidas por la IUCN, que tiene "el objetivo de contribuir a la implementación de las políticas de conservación" (UNESCO, 1995: 123). Este se ha convertido, dentro del paradigma del desarrollo sustentable, en uno de los conceptos dominantes en las políticas de conservación a nivel internacional (UNESCO, 2005).

Actualmente, la Red Mundial de Reservas de la Biósfera enlista 669 sitios en 120 países (UNESCO, 2016). La estructura intergubernamental de la UNESCO, a través del Consejo Internacional de Coordinación, principal órgano rector del MAB, proporciona a las reservas de la biósfera un marco para ayudar a los gobiernos de los países en la planificación y ejecución de programas de investigación y formación, prestándoles asistencia técnica y asesoramiento científico (UNESCO, 1995). Las reservas de la biósfera se constituyen por zonas núcleo, donde se llevan a cabo actividades que están estrictamente vinculadas a la conservación, por ejemplo, educativas y de investigación; luego siguen las zonas tampón o de amortiguamiento, que usualmente rodean a las zonas núcleo, donde se establecen actividades compatibles como la educación ambiental, recreación, ecoturismo y la investigación aplicada básica; y finalmente se encuentran las zonas de transición flexible, que comprenden variadas actividades (Primack \& Ros, 2002). Particularmente, en las zonas de amortiguamiento y en las de transición flexible, las comunidades locales, los organismos de gestión, los científicos, las organizaciones no gubernamentales, los grupos culturales, el sector económico y otros interesados, trabajan conjuntamente en la administración y el desarrollo sustentable del área (UNESCO, 1995).

En las zonas de amortiguamiento es donde se pueden realizar actividades alternativas que sean compatibles con las iniciativas de conservación. Estas operan con la lógica de que las poblaciones locales conservarán sus recursos cuando hay un incentivo para hacerlo, y/o cuando existen otras alternativas económicas atractivas para la explotación de recursos (UNESCO, 1995). El ecoturismo es una de las actividades económicas alternativas permitidas en la zona de amortiguamiento, ya que implica el desarrollo local con fines de conservar los recursos naturales; se considera esencialmente como un ejemplo de las estrategias de conservación contempladas en las reservas de la biósfera. Más aún, una característica propia del ecoturismo es que, al ser una de las actividades alternativas, su desarrollo y aplicación no debe sustituir a las actividades que tradicionalmente realizan los pobladores de las comunidades en las que se realiza, sino constituir una actividad adicional o alternativa.

Sin embargo, "lograr el éxito de integrar conservación y desarrollo socioeconómico a través del ecoturismo ha sido difícil" (Björk, 2007: 24) y varía de un caso a otro; esa valoración, constituye una parte fundamental del problema teórico y empírico de la presente investigación. Según Björk (2007, 24), existen diversos factores que influyen de manera significativa en el desempeño de los proyectos ecoturísticos en las reservas de la biósfera, entre ellos: la escasa coordinación entre los actores, la propiedad de los negocios ecoturísticos, la participación local, la infraestructura existente, el estilo de gobernanza, así como las herramientas para gestionar el comportamiento de turistas para minimizar el impacto ecológico y fomentar la conservación (Brenner y Job, 2012).

\section{Materiales y métodos}

La presente investigación adoptó un enfoque de estudio de caso, lo que incluyó un examen profundo de las actividades de ecoturismo y conservación en cuatro grupos ejidales: Adolfo López Mateos y Ruiz Cortines en la reserva de la biósfera de Los Tuxtlas-RBLT, Veracruz, México y Koiyaki (Koiyaki Group Ranch) y Lemek/ Ol Chorro (Lemek Group Ranch) en la reserva de la biósfera de Maasai Mara-RBMM, Kenia. La selección de los casos de estudio y el tipo de muestreo correspondiente fue intencionada, es decir, un muestreo donde los contextos cumplen con los criterios que se busca estudiar, basados en una valoración previa de sus características o de sus atributos. Esta se basó en el análisis de datos secundarios y en una visita de exploración que se llevó a cabo durante los meses de noviembre de 2013 en Veracruz, México y diciembre de 2013 en Maasai Mara, Kenia. Los criterios de selección no sólo se enfocaron en la existencia de actividades turísticas basadas en la naturaleza, sino en la presencia de una amplia participación de la comunidad local en las actividades ecoturísticas o alguna estrategia de gestión que 
responde a los objetivos de conservación y desarrollo dentro del Programa el Hombre y la Biósfera, y que se considera como suficiente en la literatura académica (véase Honey, 2002, 2008; Higham, 2007; Adams \& Hutton, 2007, Brenner \& Job, 2012; Vargas del Río \& Brenner, 2013).

El diseño metodológico implicó la aplicación de métodos mixtos (cuantitativo y cualitativo), basándose en un paradigma interpretativo (Patton 1990). Los métodos clave incluyeron una encuesta cuantitativa $(n=138)$, entrevistas abiertas $(n=22), 4$ grupos de enfoque, así como, la participación y observación directa. Se aplicaron los instrumentos a los residentes locales y líderes de opinión en la comunidad. El cuestionario utilizado en la parte cuantitativa se estructuró de manera que responda al objetivo principal de la investigación: caracterizar el ecoturismo como una estrategia de conservación en las Reservas de la Biósfera. En el diseño del instrumento (una escala Likert), así como en la selección de los indicadores e ítems, se tomaron en cuenta reflexiones de estudios previos sobre el tema (Vargas del Río y Brenner, 2013; Honey, 2008; Brenner y Job, 2012). En las primeras dos secciones, A. Prácticas de conservación y B. Actitudes de Conservación, se incluyeron ítems (afirmaciones) sobre el impacto del ecoturismo y la conservación para detectar las actitudes de la comunidad local. En la última sección de la encuesta, se buscó identificar los factores que influyen en el ecoturismo como estrategia de conservación. Es importante mencionar que la conceptualización del ecoturismo abarca una amplia variedad de dimensiones y criterios analíticos, por lo tanto, la selección de los indicadores e ítems incluidos en el análisis no es conclusiva. Sin embargo, permite dar un entendimiento global de los beneficios percebidos del ecoturismo y su influencia en las actitudes hacia la conservación.

El análisis cuantitativo fue descriptivo e inferencial, utilizando el paquete estadístico para Ciencias Sociales (SPSS versión 18 - SPSS Inc. 2010). Para cada afirmación, los encuestados indicaron su nivel de concordancia (si estuvieron de acuerdo, en desacuerdo, o si el comentario les fue indiferente). A través de un análisis cuidadoso, se eligió una serie de afirmaciones que claramente reflejan las tendencias de conservación (actitudes y prácticas) en los cuatro grupos ejidales. Las afirmaciones elegidas para el análisis fueron las más claras y representativas, y se seleccionaron de manera cuidadosa para reflejar las experiencias y conciencia general de los encuestados sobre la relación entre ecoturismo y conservación. Además, se identificaron las afirmaciones cuyas respuestas difirieron sustancialmente - donde la distribución de respuestas fue diferente de un grupo a otro; esta variación fue importante porque permitió el análisis estadístico adecuado (pruebas de Chi-cuadrado) para determinar las asociaciones entre las variables dependientes (actitudes y prácticas de conservación) y las variables independientes (posibles predictoras o explicativas - los beneficios directos e indirectos del ecoturismo).

En la parte cualitativa, se utilizó la técnica llamada análisis de contenido o temático. Primero, se llevaron a cabo entrevistas que fueron grabadas, trascritas y analizadas junto con fuentes de información secundaria; también se realizaron otras entrevistas no grabadas y notas de campo mediante un proceso de asignación de códigos y recuperación de la información, mediado por una clasificación en categorías analíticas. Luego se organizaron las respuestas siguiendo los temas emergentes de los datos generados como resultado de las entrevistas. Como parte del análisis cualitativo, se identificaron patrones comunes de las respuestas y el desarrollo de temas que los reflejan. Para facilitar el análisis de la información cualitativa, se utilizó un programa de cómputo (AtlasTi) basado en la organización de la información mediante asignación de códigos y categorías de análisis. Se presentan citas textuales para apreciar mejor la variedad de las opiniones de los encuestados sobre los temas identificados.

Por último, el estudio también incluyó una amplia revisión y análisis de documentos para complementar los datos primarios. Se utilizó una variedad de métodos de investigación y fuentes de datos para aumentar la triangulación, fortaleciendo de esta manera el diseño del estudio a través de controles de validez (véase Patton, 1990).

\section{Resultados y discusión}

En esta sección se presentan los resultados obtenidos de los cuatro grupos ejidales estudiados. El análisis permite sintetizar la información, sacar conclusiones, y ofrecer recomendaciones que buscan fomentar la actividad ecoturística en las reservas de la biósfera. Para facilitar la interpretación de los resultados, se presentan las características sociodemográficas de los encuestados: género, edad, origen (si nació en la comunidad o no), nivel de educación, religión, número de hijos en la familia y de personas que viven en las hogares (véase tabla 1). 
Tabla 1: Las características demográficas de los encuestados

\begin{tabular}{|c|c|c|c|c|c|c|c|}
\hline $\begin{array}{l}\text { Característica } \\
\text { demográfica }\end{array}$ & Categoría & Frecuencia & Porcentaje & $\begin{array}{c}\text { Característica } \\
\text { demográfica }\end{array}$ & Categoría & Frecuencia & Porcentaje \\
\hline \multirow{2}{*}{ Género } & Mujer & 50 & $36.2 \%$ & \multirow{2}{*}{ Religión } & $\begin{array}{l}\text { Tiene } \\
\text { religión }\end{array}$ & 121 & $88 \%$ \\
\hline & Hombre & 88 & $63.8 \%$ & & $\begin{array}{l}\text { No tiene } \\
\text { religión }\end{array}$ & 17 & $12 \%$ \\
\hline \multirow{4}{*}{$\begin{array}{l}\text { Nivel de } \\
\text { educación }\end{array}$} & $\begin{array}{l}\text { Ninguna } \\
\text { Educación }\end{array}$ & 37 & $27.1 \%$ & \multirow{3}{*}{$\begin{array}{l}\text { Número de } \\
\text { hijos en la } \\
\text { familia }\end{array}$} & 2 o menos & 38 & $27.7 \%$ \\
\hline & $\begin{array}{l}6 \text { años o } \\
\text { menos }\end{array}$ & 31 & $22.8 \%$ & & $3-5$ & 80 & $57.8 \%$ \\
\hline & 7-12 años & 50 & $36.5 \%$ & & 6 o más & 20 & $14.5 \%$ \\
\hline & $\begin{array}{l}\text { Más de } 12 \\
\text { años }\end{array}$ & 20 & $14.9 \%$ & \multirow{2}{*}{$\begin{array}{l}\text { Origen (si } \\
\text { nació en la } \\
\text { comunidad } \\
\text { o no) }\end{array}$} & Sí & 131 & $95 \%$ \\
\hline \multirow{4}{*}{ Edad } & $\begin{array}{l}30 \text { años o } \\
\text { menos }\end{array}$ & 33 & $24.0 \%$ & & No & 7 & $5 \%$ \\
\hline & $31-44$ & 27 & $19.6 \%$ & \multirow{3}{*}{$\begin{array}{l}\text { Número de } \\
\text { personas que } \\
\text { viven en los } \\
\text { hogares. }\end{array}$} & 3 o menos & 33 & $23.9 \%$ \\
\hline & $45-65$ & 46 & $33.3 \%$ & & $4-8$ & 90 & $65.2 \%$ \\
\hline & Más de 65 & 32 & $23.1 \%$ & & 9 o más & 15 & $10.9 \%$ \\
\hline
\end{tabular}

En la Tabla 2, se presenta una interpretación concisa de los resultados generados de las pruebas de Chi-cuadrado. Las afirmaciones de conservación se encuentran en la columna del lado izquierdo, mientras que las columnas del lado derecho (las últimas once columnas) representan los diferentes predictores potenciales de actitudes y prácticas de conservación, y son las variables independientes que incluyen; la situación económica ${ }^{2}$, edad, niveles de educación así como la distribución de beneficios generados de la actividad ecoturística. Se realizó una correlación entre las afirmaciones de conservación que aparecen en el lado izquierdo de la tabla y las variables predictoras que están en el lado derecho, con el fin de determinar la significación estadística en tres niveles del valor $\mathrm{p}$ $(.05, .01$ ó .001).

La segunda columna de la Tabla 2 presenta la respuesta que generalmente se esperaría de una persona que tiene una actitud positiva hacia la conservación. Por ejemplo, se espera que los miembros de la comunidad que están conscientes de la importancia del cuidado, protección y conservación del medio ambiente o que generan beneficios directos o indirectos del ecoturismo, no estén de acuerdo con la siguiente afirmación: "Debería ser permitido para los miembros de la comunidad local cortar árboles para cultivar o practicar la ganadería sin ninguna restricción de las autoridades". Este supuesto se basa en lo que señalan algunos casos reportados en la literatura previa (véase por ejemplo, Xu, Chen, $\mathrm{Lu}$ y $\mathrm{Fu}, 2006$ ). La primera fila indica las tendencias de conservación que se esperan (o son probables) al correlacionar predictores potenciales (variables independientes en las columnas del lado derecho) y las diferentes afirmaciones de conservación que aparecen en la primera columna del lado izquierdo. Las celdas con "ACE" representan actitudes de conservación esperadas, mientras "ACI" representan actitudes de conservación inesperadas y "STC" indica falta de una tendencia clara. Los niveles de significación se representan de la manera siguiente: “*” se refiere a p <.05, “**”" se refiere a p<.01 y “***” se refiere a $\mathrm{p}<.001$. 


\section{Tabla 2: Resumen de tendencias/asociaciones entre las actitudes y prácticas de conservación y predictores posibles}

\begin{tabular}{|c|c|c|c|c|c|c|c|c|c|}
\hline & & & & & Beneficios : & enerados de l & actividad $\mathrm{e}$ & coturística & \\
\hline & $\begin{array}{l}\text { La res- } \\
\text { puesta que } \\
\text { general- } \\
\text { mente se } \\
\text { esperaría }\end{array}$ & $\begin{array}{c}\text { Situación } \\
\text { económica } \\
\text { de los } \\
\text { encuestados }\end{array}$ & $\begin{array}{c}\text { Bienestar } \\
\text { de los } \\
\text { encuestados }\end{array}$ & $\begin{array}{l}\text { Situación } \\
\text { del empleo }\end{array}$ & $\begin{array}{l}\text { Responsabili- } \\
\text { dad en la } \\
\text { actividad } \\
\text { ecoturística }\end{array}$ & $\begin{array}{c}\text { Ingresos } \\
\text { indirectos } \\
\text { del } \\
\text { ecoturismo }\end{array}$ & $\begin{array}{l}\text { Intercam- } \\
\text {-bio de } \\
\text { ideas con } \\
\text { turistas }\end{array}$ & $\begin{array}{l}\text { Formación } \\
\text { y capacita- } \\
\text { ción }\end{array}$ & $\begin{array}{l}\text { Beneficios } \\
\text { de la } \\
\text { Infraes- } \\
\text { tructura }\end{array}$ \\
\hline $\begin{array}{l}\text { Las tendencias de } \\
\text { conservación que } \\
\text { se esperan (o son } \\
\text { probables). } \\
\text { Actitud de } \\
\text { conservación más } \\
\text { probable con: }\end{array}$ & & $\begin{array}{c}\text { Una } \\
\text { situación } \\
\text { económica } \\
\text { elevada }\end{array}$ & $\begin{array}{l}\text { Un mejor } \\
\text { bienestar }\end{array}$ & $\begin{array}{l}\text { Miembros } \\
\text { de la } \\
\text { familia con } \\
\text { empleo en } \\
\text { ecoturismo }\end{array}$ & $\begin{array}{c}\text { Tiene una } \\
\text { responsabili- } \\
\text {-dad } \\
\text { elevada en } \\
\text { la actividad } \\
\text { ecoturística }\end{array}$ & $\begin{array}{c}\text { Genera } \\
\text { ingresos } \\
\text { indirectos } \\
\text { del } \\
\text { ecoturismo }\end{array}$ & $\begin{array}{c}\text { Interactúa } \\
e \\
\text { intercam- } \\
\text {-bia } \\
\text { ideas con } \\
\text { turistas }\end{array}$ & $\begin{array}{l}\text { Genera be- } \\
\text { neficios de } \\
\text { formación } \\
\text { y capaci- } \\
\text { tación a } \\
\text { través del } \\
\text { ecoturismo }\end{array}$ & $\begin{array}{l}\text { Genera } \\
\text { beneficios } \\
\text { relacio- } \\
\text {-nados con } \\
\text { la mejora } \\
\text { de la } \\
\text { infraestruc- } \\
\text {-tura }\end{array}$ \\
\hline $\begin{array}{l}\text { Más que para otra } \\
\text { cosa, los recursos } \\
\text { naturales } \\
\text { (flora y fauna) } \\
\text { existen para ser } \\
\text { aprovechados } \\
\text { (leña, madera, } \\
\text { carne, etcétera). }\end{array}$ & $\begin{array}{c}\text { En } \\
\text { desacuerdo }\end{array}$ & $\mathrm{ACE}$ ** & $\mathrm{ACE}$ & STC & $\mathrm{ACE}$ & $\mathrm{ACE}$ * & $\mathrm{ACE} * *$ & ACE & $\mathrm{ACE}$ \\
\hline $\begin{array}{l}\text { A veces es } \\
\text { necesario cortar } \\
\text { árboles para } \\
\text { fines económicos } \\
\text { cuando uno no } \\
\text { tiene empleo. }\end{array}$ & $\begin{array}{c}\text { En } \\
\text { desacuerdo }\end{array}$ & $\mathrm{ACE}$ * & STC & STC & $\mathrm{ACE}$ & STC & ACE & $\mathrm{ACE}$ & STC \\
\hline $\begin{array}{l}\text { A veces es } \\
\text { necesario cazar } \\
\text { animales cuando } \\
\text { uno no tiene } \\
\text { empleo. }\end{array}$ & $\begin{array}{c}\text { En } \\
\text { desacuerdo }\end{array}$ & $\mathrm{ACE}$ & $\mathrm{ACE}$ & $\mathrm{ACI} * * *$ & $\mathrm{ACE}$ * & STC & $\mathrm{ACE}$ & $\mathrm{ACE}$ * & $\mathrm{ACE}$ * \\
\hline $\begin{array}{l}\text { Debería ser } \\
\text { permitido para los } \\
\text { miembros de la } \\
\text { comunidad local } \\
\text { cortar árboles } \\
\text { para cultivar } \\
\text { o practicar } \\
\text { la ganadería } \\
\text { sin ninguna } \\
\text { restricción de las } \\
\text { autoridades. }\end{array}$ & $\begin{array}{c}\text { En } \\
\text { desacuerdo }\end{array}$ & $\mathrm{ACE}$ & $\mathrm{ACE}$ & $\mathrm{ACE}$ * & STC & $\mathrm{ACE}$ * & $\mathrm{ACE}$ ** & $\mathrm{ACE}$ * & $\mathrm{ACE}$ ** \\
\hline $\begin{array}{l}\text { Si no fuera por } \\
\text { la conservación } \\
\text { de la reserva, } \\
\text { habría más } \\
\text { oportunidades de } \\
\text { generar ingresos. }\end{array}$ & $\begin{array}{c}\text { En } \\
\text { desacuerdo }\end{array}$ & $\mathrm{ACE}$ & $\mathrm{ACE}$ & $\mathrm{ACE}$ & STC & $\mathrm{ACE}$ * & $\mathrm{ACE} * * *$ & $\mathrm{ACE}$ ** & $\mathrm{ACE}$ ** \\
\hline $\begin{array}{l}\text { La caza de } \\
\text { animales } \\
\text { silvestres se } \\
\text { considera buena } \\
\text { cuando uno } \\
\text { necesita usarla } \\
\text { para generar } \\
\text { ingresos. }\end{array}$ & $\begin{array}{c}\text { En } \\
\text { desacuerdo }\end{array}$ & $\mathrm{ACE}$ & STC & ACI & $\mathrm{ACE}$ & STC & $\mathrm{ACE}$ * & STC & STC \\
\hline $\begin{array}{l}\text { Se involucraría } \\
\text { en prácticas de } \\
\text { uso intensivo de } \\
\text { recursos, en caso } \\
\text { de ganar más } \\
\text { dinero, sea de la } \\
\text { actividad turística } \\
\text { u otra actividad } \\
\text { económica. }\end{array}$ & $\begin{array}{c}\text { En } \\
\text { desacuerdo }\end{array}$ & STC & STC & $\mathrm{ACE}$ & $\mathrm{ACE}$ & $\mathrm{ACE}$ & $\mathrm{ACE} * * *$ & $\mathrm{ACE} * * *$ & $\mathrm{ACE} * *$ \\
\hline
\end{tabular}




\begin{tabular}{|c|c|c|c|c|c|c|c|c|c|}
\hline $\begin{array}{l}\text { El gobierno no } \\
\text { debe preocuparse } \\
\text { más por la } \\
\text { conservación } \\
\text { porque los seres } \\
\text { humanos tienen } \\
\text { más derecho } \\
\text { de vivir que los } \\
\text { animales. }\end{array}$ & $\underset{\text { En }}{\text { Eesacuerdo }}$ & $\mathrm{ACE}$ & $\mathrm{ACE}$ & STC & STC & STC & STC & $\mathrm{ACE}$ * & STC \\
\hline $\begin{array}{l}\text { Si la población de } \\
\text { animales fuera } \\
\text { alta, estaría bien } \\
\text { la caza. }\end{array}$ & $\begin{array}{c}\text { En } \\
\text { desacuerdo }\end{array}$ & STC & ACI & $\mathrm{ACE}$ & STC & STC & ACE & $\mathrm{ACE}$ & ACE \\
\hline $\begin{array}{l}\text { No es bueno cazar } \\
\text { animales salvajes. }\end{array}$ & De acuerdo & STC & ACI & STC & STC & STC & STC & STC & STC \\
\hline $\begin{array}{l}\text { Es importante } \\
\text { cuidar la flora y la } \\
\text { fauna para atraer } \\
\text { el turismo. }\end{array}$ & $\begin{array}{c}\text { En } \\
\text { desacuerdo }\end{array}$ & $\mathrm{ACE}$ & $\mathrm{ACE}$ & $\mathrm{ACE}$ & STC & ACE & $\mathrm{ACE}$ & $\mathrm{ACE}$ & STC \\
\hline $\begin{array}{l}\text { El beneficio más } \\
\text { importante de } \\
\text { la reserva es el } \\
\text { turismo. }\end{array}$ & $\begin{array}{c}\text { En } \\
\text { desacuerdo }\end{array}$ & $\mathrm{ACE}$ * & ACE & $\mathrm{ACI}$ & $\mathrm{ACE}$ & ACE & $\mathrm{ACE}$ ** & STC & $\mathrm{ACE}$ * \\
\hline $\begin{array}{l}\text { Los bosques } \\
\text { existen } \\
\text { únicamente para } \\
\text { ser aprovechados } \\
\text { de manera } \\
\text { económica. }\end{array}$ & $\begin{array}{c}\text { En } \\
\text { desacuerdo }\end{array}$ & $\mathrm{ACE} * * *$ & $\mathrm{ACE}$ & ACI & STC & STC & ACE & STC & ACE \\
\hline $\begin{array}{l}\text { Codificación: Las ce } \\
\text { "STC" indica falta d } \\
\text { refiere a } p<.01 \text { y “* } \\
\text { La muestra para to }\end{array}$ & $\begin{array}{l}\text { ldas con "AC } \\
\text { e una tenden } \\
* \text { " se refiere } \\
\text { das las comu }\end{array}$ & $\begin{array}{l}\text { represent } \\
\text { clara. Lc } \\
<.001 \text {. } \\
\text { ades es d }\end{array}$ & $\begin{array}{l}\text { titude: } \\
38\end{array}$ & serva & ada & is "A & resenta & $\begin{array}{l}\text { udes ine } \\
\text { fiere a }\end{array}$ & das y \\
\hline
\end{tabular}

En la Tabla 3, se presentan los resultados de la prueba Chi-cuadrado con más detalle, mostrando con claridad la base de las decisiones o interpretaciones presentadas en la Tabla 2. Sólo se incluyeron resultados cuyas relaciones fueron estadísticamente significativas o casi significativo tomando valor $p$ como $(<.01)$.

\section{Tabla 3: Resumen de las pruebas Chi-cuadrado entre las actitudes y prácticas de conservación y predictores posibles}

\begin{tabular}{|c|c|c|c|c|c|c|}
\hline \multirow{2}{*}{$\begin{array}{l}\text { Afirmaciones cuyas asociaciones fueron } \\
\text { estadísticamente significativas }\end{array}$} & \multicolumn{6}{|c|}{$\begin{array}{l}\text { A. } 1^{\circ} \text { Grupo (Predictor potencial); B. En desacuerdo; C. } 2^{\circ} \text { Grupo (Predictor } \\
\text { potencial); D. En desacuerdo; E. Valores p } \text { p }^{\mathrm{a}} \text {; F. Niveles de significancia }\end{array}$} \\
\hline & A. & B. & C. & D. & E. & F. \\
\hline & \multicolumn{6}{|c|}{ Situación Económica de los encuestados ${ }^{b}$} \\
\hline \multirow{2}{*}{$\begin{array}{l}\text { Más que para otra cosa, los recursos naturales (flora y } \\
\text { fauna) existen para ser aprovechados (leña, madera, } \\
\text { carne, etcétera). }\end{array}$} & Mejor & $82 \%$ & Peor & $45 \%$ & 0.0045 & $* *$ \\
\hline & Entre lo Normal & $67 \%$ & Peor & $45 \%$ & 0.0735 & \\
\hline $\begin{array}{l}\text { A veces es necesario cortar árboles para fines } \\
\text { económicos cuando uno no tiene empleo. }\end{array}$ & Mejor & $55 \%$ & Entre lo Normal & $36 \%$ & 0.0162 & * \\
\hline $\begin{array}{l}\text { El beneficio más importante de la reserva es el } \\
\text { turismo. }\end{array}$ & Entre lo Normal & $56 \%$ & Peor & $7 \%$ & 0.0164 & * \\
\hline \multirow{2}{*}{$\begin{array}{l}\text { Los bosques existen únicamente para ser } \\
\text { aprovechados económicamente. }\end{array}$} & Entre lo Normal & $90 \%$ & Peor & $59 \%$ & 0.0010 & $* * *$ \\
\hline & Peor & $77 \%$ & Peor & $59 \%$ & 0.0174 & * \\
\hline & \multicolumn{6}{|c|}{ Situación del empleo en ecoturismo } \\
\hline \multirow{2}{*}{$\begin{array}{l}\text { A veces es necesario cazar animales cuando uno no } \\
\text { tiene empleo. }\end{array}$} & $\begin{array}{l}\text { Sin empleo, } \\
\text { Sin exposición }\end{array}$ & $80 \%$ & $\begin{array}{l}\text { Sin empleo, con } \\
\text { exposición }\end{array}$ & $59 \%$ & 0.0002 & $* * *$ \\
\hline & $\begin{array}{l}\text { Sin empleo, } \\
\text { Sin exposición }\end{array}$ & $80 \%$ & Con empleo & $65 \%$ & 0.1032 & \\
\hline $\begin{array}{l}\text { Debería ser permitido para los miembros de la } \\
\text { comunidad local cortar árboles para cultivar o } \\
\text { practicar la ganadería sin ninguna restricción de las } \\
\text { autoridades. }\end{array}$ & Con empleo & $79 \%$ & $\begin{array}{l}\text { Sin empleo, con } \\
\text { exposición }\end{array}$ & $56 \%$ & 0.0182 & * \\
\hline
\end{tabular}




\begin{tabular}{|c|c|c|c|c|c|c|}
\hline \multicolumn{7}{|c|}{ Responsabilidad en la actividad ecoturística } \\
\hline $\begin{array}{l}\text { A veces es necesario cazar animales cuando uno no } \\
\text { tiene empleo. }\end{array}$ & Alta & $80 \%$ & Baja & $56 \%$ & 0.0457 & * \\
\hline \multicolumn{7}{|c|}{ Ingresos indirectos del ecoturismo } \\
\hline $\begin{array}{l}\text { Más que para otra cosa, los recursos naturales } \\
\text { (flora y fauna) existen para ser aprovechados (leña, } \\
\text { madera, carne, etcétera). }\end{array}$ & Con beneficio & $78 \%$ & Sin beneficio & $58 \%$ & 0.0476 & * \\
\hline $\begin{array}{l}\text { Debería ser permitido para los miembros de la } \\
\text { comunidad local cortar árboles para cultivar o } \\
\text { practicar la ganadería sin ninguna restricción de las } \\
\text { autoridades. }\end{array}$ & Con beneficio & $83 \%$ & Sin beneficio & $59 \%$ & 0.0208 & * \\
\hline $\begin{array}{l}\text { Si no fuera por la conservación de la reserva, habría } \\
\text { más oportunidad de generar ingresos. }\end{array}$ & Con beneficio & $75 \%$ & Sin beneficio & $47 \%$ & 0.0117 & * \\
\hline \multicolumn{7}{|c|}{ Interacción e intercambio de ideas con los turistas } \\
\hline $\begin{array}{l}\text { Más que para otra cosa, los recursos naturales } \\
\text { (flora y fauna) existen para ser aprovechados (leña, } \\
\text { madera, carne, etcétera). }\end{array}$ & Con beneficio & $78 \%$ & Sin beneficio & $49 \%$ & 0.0026 & ** \\
\hline $\begin{array}{l}\text { Debería ser permitido a los miembros de la } \\
\text { comunidad local cortar árboles para cultivar o } \\
\text { practicar la ganadería sin ninguna restricción de las } \\
\text { autoridades. }\end{array}$ & Con beneficio & $81 \%$ & Sin beneficio & $51 \%$ & 0.0012 & ** \\
\hline $\begin{array}{l}\text { Si no fuera por la conservación de la reserva, habría } \\
\text { más oportunidades de generar ingresos. }\end{array}$ & Con beneficio & $72 \%$ & Sin beneficio & $38 \%$ & 0.0001 & $* * *$ \\
\hline $\begin{array}{l}\text { La caza de animales silvestres se considera buena } \\
\text { cuando uno necesita usarla para generar ingresos. }\end{array}$ & Con beneficio & $88 \%$ & Sin beneficio & $72 \%$ & 0.0338 & * \\
\hline $\begin{array}{l}\text { Se involucraría en prácticas de uso intensivo de } \\
\text { recursos, en caso de ganar más dinero, sea de la } \\
\text { actividad turística u otra actividad económica }\end{array}$ & Con beneficio & $32 \%$ & Sin beneficio & $4 \%$ & 0.0001 & $* * *$ \\
\hline $\begin{array}{l}\text { El beneficio más importante de la reserva es el } \\
\text { turismo. }\end{array}$ & Con beneficio & $46 \%$ & Sin beneficio & $8 \%$ & 0.0056 & ** \\
\hline \multicolumn{7}{|c|}{ Formación y capacitación a través del ecoturismo } \\
\hline $\begin{array}{l}\text { A veces es necesario cazar animales cuando uno no } \\
\text { tiene empleo. }\end{array}$ & Con beneficio & $76 \%$ & Sin beneficio & $50 \%$ & 0.0141 & * \\
\hline $\begin{array}{l}\text { Debería ser permitido a los miembros de la } \\
\text { comunidad local cortar árboles para cultivar o } \\
\text { practicar la ganadería sin ninguna restricción de las } \\
\text { autoridades. }\end{array}$ & Con beneficio & $86 \%$ & Sin beneficio & $60 \%$ & 0.0125 & * \\
\hline $\begin{array}{l}\text { Si no fuera por la conservación de la reserva, habría } \\
\text { más oportunidades de generar ingresos. }\end{array}$ & Con beneficio & $80 \%$ & Sin beneficio & $47 \%$ & 0.0025 & ** \\
\hline $\begin{array}{l}\text { Se involucraría en prácticas de uso intensivo de } \\
\text { recursos, en caso de ganar más dinero, sea de la } \\
\text { actividad turística u otra actividad económica. }\end{array}$ & Con beneficio & $41 \%$ & Sin beneficio & $11 \%$ & 0.0002 & $* * *$ \\
\hline $\begin{array}{l}\text { El gobierno no debe preocuparse más por la } \\
\text { conservación porque los seres humanos tienen más } \\
\text { derecho de vivir que los animales. }\end{array}$ & Con beneficio & $80 \%$ & Sin beneficio & $57 \%$ & 0.0321 & * \\
\hline \multicolumn{7}{|c|}{ Beneficios de la infraestructura } \\
\hline $\begin{array}{l}\text { A veces es necesario cazar animales cuando uno no } \\
\text { tiene empleo. }\end{array}$ & Con beneficio & $68 \%$ & Sin beneficio & $46 \%$ & 0.0404 & * \\
\hline $\begin{array}{l}\text { Debería ser permitido para los miembros de la } \\
\text { comunidad local cortar árboles para cultivar o } \\
\text { practicar la ganadería sin ninguna restricción de las } \\
\text { autoridades. }\end{array}$ & Con beneficio & $82 \%$ & Sin beneficio & $53 \%$ & 0.0018 & ** \\
\hline $\begin{array}{l}\text { Si no fuera por la conservación de la reserva, habría } \\
\text { más oportunidades de generar ingresos. }\end{array}$ & Con beneficio & $68 \%$ & Sin beneficio & $45 \%$ & 0.0076 & ** \\
\hline $\begin{array}{l}\text { Se involucraría en prácticas de uso intensivo de } \\
\text { recursos, en caso de ganar más dinero, sea de la } \\
\text { actividad turística u otra actividad económica }\end{array}$ & Con beneficio & $30 \%$ & Sin beneficio & $9 \%$ & 0.0027 & ** \\
\hline $\begin{array}{l}\text { El beneficio más importante de la reserva es el } \\
\text { turismo. }\end{array}$ & Con beneficio & $40 \%$ & Sin beneficio & $29 \%$ & 0.0206 & * \\
\hline
\end{tabular}




\subsection{Situación económica como predictora de las actitudes y prácticas sobre la conservación}

Los resultados demuestran una asociación significativa entre empleo en la actividad ecoturística y las prácticas de conservación; por otro lado, la influencia del ecoturismo en las actitudes de conservación parece ser estadísticamente insignificante. Generalmente, beneficios indirectos del ecoturismo como interacción e intercambio de ideas con ecoturistas, y formación y capacitación, mostraron asociaciones o tendencias claras y positivas en cuanto a las actitudes de conservación. Por lo tanto, se reconoce que los beneficios indirectos, por ejemplo la participación local en las actividades del ecoturismo y distribución de ingresos, son los factores más importantes porque determinan actitudes y prácticas positivas de las comunidades locales hacia el medio ambiente. Es importante mencionar que los beneficios del ecoturismo van más allá del ingreso económico directo, se deben contemplar estrategias para asegurar ambos beneficios (directos e indirectos). Por ejemplo, llevar a cabo talleres y cursos con la participación de las diferentes partes interesadas para fomentar el conocimiento y las capacidades interpretativas del medio ambiente.

Una correlación entre la situación económica y las actitudes de conservación puede revelar diferentes situaciones. Como se muestra en las Tablas 2 y 3 , las pruebas Chi-cuadrado generaron cuatro aspectos de la situación económica con tendencias claras y significativas, mientras otros aspectos no expresaron ninguna tendencia clara. En términos generales, los encuestados que dijeron tener un mejor bienestar económico expresaron actitudes positivas hacia la conservación, aunque en algunos casos, quienes percibieron su situación como normal, mostraron actitudes de conservación más positivas. Basado en estos resultados, se concluye que los encuestados cuya situación económica es baja (por falta de beneficios del ecoturismo), tienen actitudes negativas de conservación, en contraste con aquellos cuya situación económica es alta. Uno de los entrevistados comentó:

Antes, yo pensaba que no se podría generar nada de los bosques, ahora sí, entiendo que el cuidado de los bosques es importante para el turismo... mi ingreso depende directamente de los turistas, cuando vienen más turistas puedo generar más dinero de mi trabajo, de una manera u otra, las llegadas de turistas dependen del estado de cuidado de la reserva.

Sin embargo, uno de los hallazgos más sorprendentes es que existe una asociación entre la situación económica y la motivación para involucrarse o participar en prácticas de uso intensivo de recursos, (esta pregunta abarcó conductas dañinas al medio ambiente, como por ejemplo, deforestación, compra de maquinaria para incrementar la actividad agrícola, compra de más ganado, etcétera). El hallazgo se considera interesante porque contradice la expectativa nomológica que establece que las mejoras del bienestar económico de las comunidades locales disminuyen prácticas que empeoran el estado del medio ambiente. En este sentido, se acepta la primera hipótesis: que por sí solos los incentivos económicos no son suficientes para que las comunidades tengan prácticas y actitudes positivas hacia la conservación. Se contradicen también los hallazgos de Honey (2008), que encontró una asociación positiva entre altos niveles de bienestar económico y mayor porcentaje de terrenos dedicados a la conservación en las comunidades locales; en su trabajo, la autora concluye que el aumento en ingresos a través de actividades económicas alternativas como el ecoturismo, automáticamente motiva a los miembros de la comunidad local para asignar más terrenos a las necesidades de conservación y protección de la biodiversidad (por ejemplo, repoblación forestal y cuidado de especies en peligro de extinción).

Sin embargo, diferentes autores advierten contra la dependencia económica de los recursos naturales como una estrategia para impulsar la conservación. Xu et al. (2006) señalan que demasiado énfasis en el aprovechamiento económico de las reservas de la biósfera crea una falsa noción por parte del liderazgo, de que las áreas naturales protegidas pueden conservarse con éxito sólo cuando se garantizan beneficios económicos para las comunidades locales. Los resultados generados de las entrevistas confirman esta preocupación, dado que los entrevistados que dependen de los recursos naturales para su bienestar económico (a través del ecoturismo o venta de madera) identificaron a los beneficios económicos como su motivación principal para conservar la biodiversidad. Boley y Green (2016) por su parte, demostraron que la dependencia económica así como el uso excesivo de los recursos naturales (normalmente desfasado como uso alternativo), puede causar degradación del medio ambiente y limitar la sustentabilidad a largo plazo de estos. Otros autores (véase Tang, Gao y Shi, 2015 y Chatty y Colchester, 2002) enfatizan la necesidad de influir valores de conservación entre los miembros de la comunidad como una estrategia alternativa (y más viable) para cambiar los comportamientos y las actitudes no deseables hacia el medio ambiente. Según estos autores, los beneficios económicos son incentivos a corto plazo que realmente no logran cambiar la forma en que los miembros de la comunidad piensan y actúan; cuando los beneficios generados de las reservas disminuyen o desaparecen, es posible que vuelvan a usar los recursos naturales de forma destructiva, si es que lo hacían antes. 


\subsection{Beneficios indirectos del ecoturismo como predictores de prácticas y actitudes hacia la conservación}

Los resultados demuestran que los beneficios indirectos del ecoturismo generan actitudes de conservación positivas. En las Tablas 2 y 3, se observa que los encuestados con beneficios indirectos, por ejemplo: ingresos indirectos, interacción e intercambio de ideas con turistas, capacitación, formación y mejoramiento de la infraestructura, respondieron de manera favorable a las afirmaciones sobre la conservación. También estuvieron claramente en desacuerdo con la afirmación: "se involucraría en prácticas de uso intensivo de recursos, en caso de ganar más dinero, sea de la actividad turística u otra actividad económica”, cuando se les preguntó qué harían con su dinero si tuvieran más. En general, se encontró que todas las asociaciones/correlaciones referentes a los beneficios indirectos del ecoturismo y las actitudes hacia la conservación generaron valores estadísticamente significativos, con la excepción de ingresos indirectos; además, dichas asociaciones fueron positivas. Al menos tres de los cuatro indicadores del beneficio indirecto mostraron una asociación positiva con las afirmaciones sobre la conservación; asimismo se encontró una alta significación estadística para algunas de las asociaciones (véase Tabla 3). Estos hallazgos confirman las siguientes hipótesis: 1) Los incentivos económicos por sí sólos no son suficientes para que las comunidades locales tengan actitudes y prácticas que favorezcan la conservación, otros factores como distribución de beneficios, involucramiento y empoderamiento motivan e influyen en las actitudes y prácticas hacia la conservación, y 2) Una mayor participación de las comunidades locales en las actividades del ecoturismo pueden posiblemente generar actitudes y prácticas positivas que apoyan a la conservación.

Los hallazgos del presente estudio coinciden con los de Tran y Walter (2014) quienes señalan que los beneficios indirectos tales como mejoramiento de la infraestructura, empoderamiento de la comunidad local, y la formación y capacitación, son más importantes y tienen una asociación más fuerte con las actitudes de conservación que los beneficios directos, por ejemplo, empleo o beneficios monetarios. Los cuatro indicadores de beneficios indirectos en el presente estudio mostraron asociaciones estadísticamente significativas y positivas con las siguientes afirmaciones sobre la conservación (véase Tabla 3): "debería ser permitido para los miembros de la comunidad local cortar árboles para cultivar o practicar la ganadería sin ninguna restricción de las autoridades" y "si no fuera por la conservación de la reserva, habría más oportunidades de generar ingresos". Es interesante señalar que el empleo como factor predictor no mostró una relación estadísticamente significativa con las dos afirmaciones anteriores, a pesar de que los encuestados mencionaron con frecuencia durante las entrevistas que son beneficiarios del empleo en la industria ecoturística gracias a las iniciativas de conservación realizadas por las diferentes partes interesadas (el gobierno, la comunidad local, entre otras).

Entre los cuatro indicadores de beneficios indirectos como predictores de actitudes, el empleo mostró el menor número de asociaciones significativas con actitudes positivas de conservación, por otra parte, el intercambio de ideas con turistas reveló el mayor número de asociaciones significativas con actitudes positivas sobre la conservación (véase las Tablas 2 y 3). La capacitación y mejoramiento de la infraestructura como factores predictores de las actitudes sobre la conservación propiciaron asociaciones parecidas a lo que se encontró sobre la interacción e intercambio de ideas con turistas. Es evidente que los encuestados que generan beneficios indirectos interactuando con los ecoturistas, tienen actitudes positivas hacia la conservación; la misma tendencia se encontró en casi todas las afirmaciones que representaron las actitudes de conservación, de hecho, la mitad de las asociaciones fueron estadísticamente significativas y en la mayoría, la significación estadística fue alta. Por lo tanto, se puede concluir que las interacciones directas entre los miembros de la comunidad local y los ecoturistas forman una consideración importante para los que buscan crear o fortalecer actitudes positivas hacia el medio ambiente en zonas adyacentes a las reservas de la biósfera. En las entrevistas, uno de los guías turísticos comentó:

Para mí, el turismo es bueno. Me ha dado la oportunidad de interactuar con personas de culturas diferentes, yo nací aquí y he vivido aquí toda mi vida, por eso, creo que el turismo es bueno para la comunidad. Cuando hay grandes grupos de turistas, hacemos la cena aquí, e invitamos a varios vecinos para convivir, tocamos instrumentos, cantamos e interactuamos con los turistas, de hecho algunos de nosotros han recibido muchísimos regalos de los turistas.

Se encontró también que el intercambio de ideas (53\%) es el beneficio más popular del ecoturismo en las cuatro comunidades. Es importante recordar que entre todas las variables aplicadas para predecir las tendencias de conservación, los beneficios indirectos del ecoturismo mostraron el mayor número de tendencias estadísticamente significativas. Por lo tanto, es posible concluir con base en los hallazgos que 
los beneficios indirectos son los predictores más influyentes a las actitudes y prácticas de conservación, sin embargo, es necesario realizar más estudios en profundidad para calificar/categorizar diferentes beneficios del ecoturismo (directos e indirectos), según su importancia, y examinar su confiabilidad como predictores de actitudes y prácticas de conservación. Probablemente se tendrán que incluir más grupos ejidales, diferentes a los incluidos en el presente estudio.

\section{Conclusiones}

Esta investigación presenta un panorama completo sobre la eficacia del ecoturismo como una estrategía de conservación en las Reservas de la Biósfera. Resultados de los cuatro grupos ejidales ilustran dos conclusiones importantes: por un lado, se concluye que el ecoturismo puede generar importantes beneficios económicos para las comunidades locales e incentivar su participación en iniciativas de conservación, evitando prácticas destructivas, como la tala de árboles, la cacería o actividades que implican uso intensivo de los recursos naturales como la agricultura. Cabe señalar que no es siempre deseable evitar o acabar totalmente las actividades antes mencionadas, se pueden integrar las diferentes actividades socioeconómicas en los espacios rurales (las reservas de la biósfera incluidas), a través de un diseño sustentable de desarrollo que va más allá del aprovechamiento de recursos naturales para fines turísticos y tomar en cuenta la complejidad de los espacios rurales. Se destaca el ejemplo de la agroecología y la agronomía convencional que se basan en la aplicación de los conceptos y principios de la ecología al diseño, desarrollo y gestión de sistemas agrícolas sostenibles.

Por otro lado, el ecoturismo puede influir de manera positiva en las actitudes y prácticas de conservación cuando se generan dichos beneficios económicos. Además, se encontró que los incentivos económicos directos no son los únicos factores de motivación que influyen en las actitudes y prácticas de conservación. Por lo que se concluye que el ecoturismo se debe de considerar como un solo componente del plan maestro que busca lograr la conservación y el desarrollo socioeconómico a través de diferentes estrategias; el plan maestro también debe incluir otros factores como leyes y restricciones, y la participación e involucramiento de las comunidades locales en la gestión de las reservas, entre otras. Asimismo, se considera pertinente cambiar la forma de aplicar leyes y restricciones sobre el uso de recursos naturales en los alrededores de las reservas.

Se concluye también que los beneficios indirectos del ecoturismo, como la interacción e intercambio de ideas con los ecoturistas, formación y capacitación, influyen más en las actitudes y prácticas de conservación que los beneficios directos, como los ingresos generados del empleo. Esta conclusión tiene al menos dos implicaciones. En primer lugar, será mejor que los actores de la actividad ecoturística concentren sus esfuerzos fomentando los beneficios indirectos en lugar de enfocarse más en los beneficios directos; de esta manera, los negocios ecoturísticos pueden lograr más en sus iniciativas de conservación. Segundo, se debe de permitir y facilitar una mayor interacción con los turistas, que sea culturalmente apropiada, y mejorar las oportunidades de formación y capacitación sobre temas de conservación para todos los empleados.

Sería imprudente sugerir que existe un nivel de la actividad ecoturística ideal que no se considera excesivo, y ofrece máximos beneficios con mínimos impactos. Así pues, el éxito o fracaso de la actividad ecoturística puede variar en diferentes casos y depende del contexto local, además de los factores sociodemográficos, como la cultura. Por otro lado, se distingue un caso de otro por los enfoques y estrategias empleadas principalmente por los operadores ecoturísticos para integrar la conservación y el desarrollo de las comunidades. Cuando el ecoturismo ocupa un papel importante en la economía local, y ofrece beneficios sin perjudicar el bienestar de los recursos naturales que son la base económica de la comunidad, existen posibilidades de integrar la conservación y el desarrollo socioeconómico. Sin embargo, se recomiendan estudios más críticos y profundos para evaluar las relaciones de poder entre los diferentes actores y las comunidades.

\section{Bibliografia}

Adams, W. M., \& Hutton, J.

2007. People, Parks and Poverty: Political Ecology and Biodiversity Conservation. Conservation and

Society , 5(2): 147-183. URL: http://www.conservationandsociety.org/text.asp?2007/5/2/147/49228

Adams, J. S., \& McShane, T. O.

1992. The myth of wild Africa: conservation without illusion. Univ of California Press. 
Alcamo, J., \& Bennett, E. M.

2003. Ecosystems and human well-being a framework for assessment - Millenium Ecosystem Assessment

(Program), Washington, D.C.: Island Press.

Batisse, M.

1980. The relevance of MAB. Environmental Conservation, 7(3): 179-184.

Batisse, M.

1982. The biosphere reserve: a tool for environmental conservation and management. Environmental Conservation, 9(2): 101-111.

Björk, P.

2007. Definition Paradoxes: From Concept to definition. En: Higham, J. E. (Ed.) Critical Issues in Ecotourism: Understanding a complex phenomenon (pp. 23-45). Oxford, UK: Butterworth-Heinemann. Blamey, R. K.

2001. Principles of ecotourism. The encyclopedia of ecotourism, 2001, 5-22.

Boley, B. B., \& Green, G. T.

2016. Ecotourism and natural resource conservation: the 'potential' for a sustainable symbiotic relationship. Journal of Ecotourism, 15(1): 36-50. URL: http://dx.doi.org/10.1080/14724049.2015.1094080 Brenner, L., \& Job, H.

2012. Challenges to Actor-Oriented Environmental Governance: Examples from three Mexican Biosphere Reserves. Tijdschrift voor economische en sociale geografie, 103(1): 1-19. URL: http://onlinelibrary. wiley.com/doi/10.1111/j.1467-9663.2011.00671.x/full

Budowski, G.

1976. Tourism and environmental conservation: conflict, coexistence, or symbiosis? Environmental conservation, 3(1): 27-31. URL: https://doi.org/10.1017/S0376892900017707

Cardoso-Jiménez, C.

2006.Turismo Sostenible: una revisión conceptual aplicada. El Periplo Sustentable, (11), pp. 5-21. URL: http://www.redalyc.org/html/1934/193420679001/

Ceballos-Lascurain, $\mathrm{H}$.

1996. Tourism, ecotourism and protected areas, IUCN (World Conservation Union), Switzerland.

Ceballos-Lascurain, 1998, Ecoturismo, Naturaleza y Desarrollo Sostenible, Estrategia Nacional de Ecoturismo para México, Secretaría de Turismo, México D.F.

Chatty, D., \& Colchester, M.

2002. Conservation and Mobile Indigenous Peoples: Displacement, Forced Settlement and Sustainable Development. Berghahn, New York.

De La Rosa, B. M.

2003. Nuevos turistas en busca de un nuevo producto: el patrimonio cultural. Pasos. Revista de Turismo y Cultura, 1(2): 157-159. URL: http://www.pasosonline.org/Publicados/1203/PS030603.pdf

Gómez, N. S.

2011. La planificación de los destinos turísticos mexicanos: una receta mil veces vanagloriada. Renovación y Restructuración de Destinos Turísticos Consolidados del Litoral, Bloque Temático 1: Principios y fundamentos de los procesos de renovación y reestructuración en el marco del ciclo de vida de los destinos turísticos (págs. 1-21). Alicante, España: Universidad de Alicante.

Goodwin, H.

1996. In pursuit of ecotourism. Biodiversity \& Conservation, 5(3): 277-291. URL: https://link.springer. com/article/10.1007\%2FBF00051774?LI=true

Goodwin, H.

2007. Indigenous tourism and poverty reduction. En: Butler, R., \& Hinch, T. (Eds.), Tourism and indigenous peoples: Issues and implications, pp. 84-94, United Kingdom: Butterworth-Heinemann.

Guerrero Rodríguez, R.

2010. Ecoturismo Mexicano: la promesa, la realidad y el futuro. Un análisis situacional mediante estudios de caso, El Periplo Sustentable, (18), pp. 37-67. URL: http://www.redalyc.org/html/1934/193414423002/

Higham, J.

2007. Ecotourism: Competing and Conflicting Schools of thought. En J. Higham, Critical Issues in Ecotourism: Understanding a complex Phenomenon (págs. 20-40). Oxford, UK: Butterworth-Heinemann. Honey, M.

2002. Ecotourism and Certification: Setting Standards in Practice. Washington DC: Island Press. URL: https://islandpress.org/book/ecotourism-and-certification 
Honey, M.

2008. Ecotourism and Sustainable Development: Who Owns Paradise? Washington, DC.: Island Press.

URL: https://islandpress.org/book/ecotourism-and-sustainable-development-second-edition

Hvenegaard, G.

1994. Ecotourism: A Status Report and Conceptual Framework. Journal of Tourism Studies, vol. 5 (2) 24-35. URL: http://search.informit.com.au/documentSummary;dn=950706232;res=IELAPA

IUCN

1994. Guidelines for protected area management categories, International Union for the Conservation of Nature - World Monitoring Conservation Centre, Gland, Switzerland and Cambridge, UK.

Jamal, T., \& Camargo, B. A.

2014. Sustainable tourism, justice and an ethic of care: Toward the just destination. Journal of Sustainable Tourism, 22(1): 11-30. URL: https://www.tandfonline.com/doi/abs/10.1080/09669582.2013.786084 Kinker, $\mathrm{S}$.

2002. Ecoturismo e conservação da natureza em parques nacionais. Papirus Editora. Campinas, SP.

Laven, D. N., Wall-Reinius, S., \& Fredman, P.

2015. New challenges for managing sustainable tourism in protected areas: an exploratory study of the European Landscape Convention in Sweden. Society \& natural resources, 28(10), 1126-1143. URL: https://www.tandfonline.com/doi/abs/10.1080/08941920.2015.1013166?journalCode=usnr20

Magio, K.O. Velarde, M.V., Santillán, M. N. and Ríos G.

2013. Ecotourism in Developing Countries: A Critical Analysis of the Promise, the Reality and the Future Journal of Emerging Trends in Economics and Management Sciences (JETEMS), Vol. 4(5): 481-486. URL: http://jetems.scholarlinkresearch.com/articles/Ecotourism.pdf

Mc Coy Cador, C. E., \& Sosa Ferreira, A. P.

2016. Causas y efectos de un destino no sustentable: Caso playas públicas de Cancún, Quintana Roo. El periplo sustentable, (31). URL: https://rperiplo.uaemex.mx/article/view/4887/3403

Patton, A.

1990. Stretch Your Product's Earning Years: Top Management's Stake in the Product Life Cycle, Management Review, 48(6): 9-14.

Pérez Villegas, G.; Carrascal, E.

2000. El desarrollo turístico en Cancún, Quintana Roo y sus consecuencias sobre la cubierta vegetal. Investigaciones Geográficas, 43, pp. 145-166. URL: http://www.scielo.org.mx/scielo.php?script=sci_ar ttext\&pid=S0188-46112000000300010

Primack, R. B. \& Ros, J.

2002. Introducción a la biología de la conservación, Ariel, Barcelona.

Rudzewicz, L., \& Lanzar, R. M.

2008. Ecoturismo y conservación de los ecosistemas: Reservas Particulares de Patrimonio Natural en Brasil. Estudios y perspectivas en turismo, 17(3): 226-249. URL: http://www.scielo.org.ar/scielo. php?script=sci_arttext\&pid=S1851-17322008000300002

Stronza, A., \& Durham, H. W.

2008. Ecotourism and Conservation in the Americas. CAB International, Oxfordshire, UK.

Tang, L. N., Gao, L. J., \& Shi, L. Y.

2015. Special Issue: Sustainable management and protection of ecosystems with high conservation values in Shangri-La County, Yunnan Province, China. International Journal of Sustainable Development and World Ecology, 22(2), 99-188. URL: https://www.cabdirect.org/cabdirect/abstract/20153101361

Tran, L., \& Walter, P.

2014. Ecotourism, gender and development in Northern Vietnam, Annals of Tourism Research, 44: 116-130. URL: https://doi.org/10.1016/j.annals.2013.09.005

UNESCO

1995. Reservas de la biósfera: La estrategia de Sevilla y el marco estatutario de la red mundial, UNESCO, París. UNESCO

2005. Biosphere reserves benefits and opportunities, Man and Biosphere Program. Recurso electrónico http://portal.unesco.org.

UNESCO

2016. Directory of the World Network of Biosphere Reserves (WNBR), Ecological Sciences for Sustainable Development. United Nations Educational Cientific and Cultural Organization. Disponible en: http:// www.unesco.org/new/en/natural-sciences/environment/ecological-sciences/biosphere-reserves/world-network-wnbr/wnbr/ 
Van Cuong, C., Dart, P., \& Hockings, M.

2017. Biosphere reserves: Attributes for success. Journal of Environmental Management, 188, 9-17. URL: https://doi.org/10.1016/j.jenvman.2016.11.069

Vargas del Río, D., \& Brenner, L.

2013. Ecoturismo comunitario y conservación ambiental: la experiencia de La Ventanilla, Oaxaca, México. Estudios sociales (Hermosillo, Son.), 21(41): 31-63. URL: http://www.scielo.org.mx/scielo. php?script=sci_arttext\&pid=S0188-45572013000100002

$\mathrm{Xu}$, J., Chen, L., Lu, Y., \& Fu, B.

2006. Local people's perceptions as decision support for protected area management in Wolong Biosphere Reserve, China. Journal of Environmental Management, 78(4): 362-372. URL: https://doi.org/10.1016/j. jenvman.2005.05.003

\section{Notas}

1 Posteriormente, la definición se adoptó por la Unión Internacional para la Conservación de la Naturaleza - IUCN por sus siglas en inglés- (Ceballos-Lascuráin, 1998, p. 6).

2 En esta variable, se les pidió a los encuestados que evaluaran/ compararan su situación económica con otros miembros de la comunidad, es decir, "mejor", "entre lo normal" o "peor". 